\title{
À vos caddies, citoyens! La révolution, motif politique saisi par la publicité
}

\section{Caroline de Montety}

\section{(2) OpenEdition \\ 1 Journals}

Édition électronique

URL : http://journals.openedition.org/mots/20623

DOI : $10.4000 /$ mots. 20623

ISSN : 1960-6001

Éditeur

ENS Éditions

\section{Édition imprimée}

Date de publication : 1 mai 2012

Pagination : 63-78

ISBN : 978-2-84788-356-5

ISSN : 0243-6450

\section{Référence électronique}

Caroline de Montety, «À vos caddies, citoyens ! La révolution, motif politique saisi par la publicité », Mots. Les langages du politique [En ligne], 98 | 2012, mis en ligne le 01 mai 2014, consulté le 19 avril 2019. URL : http://journals.openedition.org/mots/20623 ; DOI : 10.4000/mots.20623 


\section{À vos caddies, citoyens ! La révolution, motif politique saisi par la publicité}

L'intensification des rapports entre publicité et politique invite à interroger les représentations dont se nourrissent ces deux types de discours et d'imaginaires. Nous nous focaliserons dans cet article sur les saisies du politique par la publicité, laissant volontairement de côté la dynamique inverse.

Les mécaniques d'appropriations publicitaires ne sont pas spécifiquement réductibles au champ du politique, toutes les formes sociales, médiatiques, culturelles, religieuses pouvant devenir un enjeu pour les marques. Néanmoins, elles ont, quand elles mobilisent le politique, des logiques et modalités particulières que nous tenterons de mettre au jour en observant leurs entrelacs.

Quelles sont les rhétoriques de marques autour du politique remaniant les représentations du politique et du publicitaire pour susciter l'adhésion dans les univers concurrentiels où elles sont mobilisées?

Pour les marques, mobiliser les références au politique semble une tentation d'autant plus aiguë que les représentations de la consommation ont évolué : la doxa marketing actuelle tend à considérer que la bonne communication des marques doit être «holistique » et s'adresser désormais à des consommateurs, à la fois acteurs économiques, individus, citoyens, etc. Sont ainsi apparues ces dernières années de nombreuses campagnes commerciales pour des automobiles, voyages, enseignes de distribution, radios, etc., faisant référence au politique, avec la reprise de slogans ou de thèmes politiques.

Ce qui frappe au premier regard, c'est l'importance des manifestes publicitaires fondés sur le détournement ironique, la publicité misant alors sur l'actualité et la connivence avec le destinataire des messages : «Bling-bling» et Ryan air, «Vivre ensemble» de RTL, etc.

Dans cette mise en dérision des travers de la classe politique ou de ses acteurs, la crédibilité du politique est ici mise en cause. La dépolitisation actuelle souvent évoquée facilite grandement la saisie des mots du politique et ces détournements.

Il semblerait pourtant, au second regard, qu'un nombre conséquent de 
publicités joue sur autre chose, que l'on pourrait qualifier de grandeur du politique. La perte de crédibilité du politique n'a apparemment pas détruit tout à fait l’idéal politique. La représentation de la politique comme espace de compétition et de conquête du pouvoir se double d'une représentation de la politique plus noble : l'art de gouverner pour le bien-être de la cité. En s'attachant à cet aspect, les publicitaires semblent se saisir d'une légitimité dont la publicité est en quête, dans un contexte où elle est souvent fortement critiquée et disqualifiée.

Après avoir examiné un grand nombre de publicités liées au politique, parues ces dernières années dans la presse ou sous forme d'affiches, et après avoir écarté les publicités, évoquées plus haut, se moquant du et des politiques, nous avons repéré une thématique forte : l'idéal révolutionnaire.

Le thème de la révolution est apparu de façon récurrente dans la lecture flottante de ce corpus lié au politique à travers des publicités reprenant des lexiques et syntagmes révolutionnaires : slogans de Mai 68, iconographie du poing levé, affichage de droits, etc.

C'est à partir de l'analyse de ce corpus et de ses modalités rhétoriques que nous questionnerons, à travers quelques exemples représentatifs, l’idéal révolutionnaire comme réservoir de puissance pour la publicité et les marques qu'elle promeut.

La publicité se nourrit des mythes politiques. Le mythe s'entend ici non pas dans le sens commun de fiction mais dans le sens barthésien, ni mensonge ni aveu mais «inflexion », contenant un savoir "confus, formé d'associations molles, illimitées» (Barthes, 1957, p. 204) qui va pouvoir, par cette discontinuité, être tissé avec les contingences publicitaires fondées sur les stratégies d'entreprise et les nécessités de la captation et de la crédibilité.

Cette posture pose le problème du paradoxe à étudier une saisie des signes du politique au regard des mythes alors que, selon Barthes, «le mythe est une parole dépolitisée » (ibid., p. 229). Comment la publicité, «dimension constitutive de la société bourgeoise» (Habermas, 1963), peut-elle mettre en scène l'idéal révolutionnaire?

Ce rapprochement oxymorique sera l'occasion idéale d'examiner les liens, entrelacs, glissements, convergences et paradoxes des rapprochements entre publicité et politique et d'observer le maniement des représentations du politique quand il est saisi par la matrice publicitaire.

\section{La révolution utilisée comme embrayeur consumériste}

«La France réclame du neuf », affirme Ikea, marque emblématique du «Design démocratique » dont elle a fait son slogan ; "Tous unis contre la vie chère », proclame Intermarché; "Tous simply citoyens », rappelle son concurrent Simply.

Si d'autres acteurs économiques que les enseignes de distribution adoptent 
parfois ce type de discours (par exemple Rougier et Plé et sa « révolution des prix», Nike et le «football libre»), la prépondérance de la révolution comme thème privilégié dans la grande distribution s'est imposé.

Leclerc est la marque emblématique de ce choix rhétorique avec la diffusion de deux campagnes en 2004 et 2008 . Leclerc a opté pour le détournement de certains slogans de mai 1968 en 2004 et 2005 dans une campagne contre la loi Galland ${ }^{1}$, que le distributeur voulait désigner comme responsable de la hausse des prix et qu'il a réitéré en 2008, à l'occasion des quarante ans des "événements», en s'élevant notamment contre le monopole des pharmacies.

La récurrence de la contestation dans les publicités Leclerc se manifeste avec la présence réitérée d'une imagerie très présente dans d'autres campagnes de marque : le poing levé.

\section{Modalités du poing levé : glissements...}

Ce geste est repris dans plusieurs campagnes Leclerc de 2005 comme indicateur représentatif de la posture de la marque :

- «il est interdit d'interdire de vendre moins cher»,

- «La croissance oui sauf celle des prix!»,

- "La hausse des prix oppresse votre pouvoir d'achat».

Ces trois publicités ${ }^{2}$ conçues par l'Agence Australie sont accompagnées du slogan de Leclerc : «E. Leclerc défend votre pouvoir d'achat».

Les typographies et les choix iconiques divergent, mais ces trois manifestes publicitaires mettent en scène un «poing levé», métaphore de la tension soulignée par les marques entre l'indifférence d'un partenaire aussi flou qu'implicite (pouvoirs publics, société...) et les attentes des consommateurs; c'est une métaphore d'amplification qui sursignifie cette tension ou ce désaccord.

Sans prétendre à une anthropologie historique et politique du poing levé, il semble utile de replacer ce geste dans son contexte d'apparition et de circulation. En effet, le poing levé est plus qu'une image : il est l'emblème d'un geste politique rituel historiquement marqué. L'historien Gilles Vergnon souligne les modalités de sa circulation: «Rite simple, élémentaire, qui redouble dans sa forme collective celui du mouvement spontané de menace, de colère ou de liesse, il ne nécessite aucun support “technique” et se prête à la manifestation collective comme à la pose individuelle.» (Vergnon, 2005, p. 78)

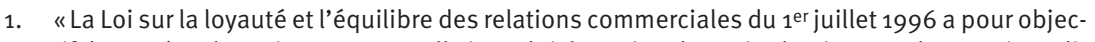
tif de protéger le petit commerce. Elle introduit la notion de «prix abusivement bas » et interdit aux grandes surfaces d'intégrer les marges arrière dans le calcul de la vente à perte. Cette loi a été modifiée par la loi Dutreil et la loi Chatel. » Source : [http://www.mercator-publicitor.fr/ lexique-marketing-definition-loi-galland].

2. Ces publicités, que nous n'avons pas été autorisée à reproduire ici, sont visibles sur le site pics. agora : [http://pics.agora.eu.org/spip.php?page=recherche\&recherche=Leclerc], consulté le 23 janvier 2012. 
C'est ainsi que ce geste, né dans les années 1930 comme geste d'opposition au geste fasciste du bras tendu, s'est répandu dans les mouvements contestataires de gauche, communistes puis socialistes. Le rite du poing levé circula ainsi du Front populaire jusqu'aux mouvements contestataires de 1968, dans les mouvements syndicaux, etc. Fortement médiatisé par la photographie, le cinéma, approprié par des individus et des collectifs, il constitue, pour le lecteur, « une invitation à la reprise mimétique, à la pose : le lever de poing face à l'objectif braqué du Leica ouvre la possibilité de se retrouver sur une photographie publiée ou dans le film d'actualités... » (id., p. 89).

Le poing levé s'est diffusé dans la culture comme figure d'une revendication légitime, d'un contre-pouvoir, d'une manifestation qui passe sous silence ce que la révolution peut avoir de plus violent : les combats, armes et corps à corps, le sang...

\section{Jeux de pouvoir}

Dans la publicité «il est interdit d'interdire de vendre moins cher», l'image représente cinq silhouettes de personnages en réserve noire sur fond rouge, détournement d'une affiche célèbre de Mai 68, «nous sommes le pouvoir»3, représentant six ouvriers (interprétés comme tels en vertu d'une clé portée par le personnage de gauche).

Les personnages de la publicité Leclerc, trois hommes et deux femmes, sont de natures différentes : les trois personnages centraux sont statutairement indéfinis, la femme à gauche tient de sa main droite un caddie de courses rempli tandis que l'homme à droite lève son poing gauche. L'outil emblématique de l'ouvrier sur l'affiche originale est remplacé par le caddie de la ménagère. Le rapport texte-image suggère un parcours narratif conduisant d'une figure à l'autre, le lien implicite pouvant être reconstruit comme la cherté des produits.

Le chariot de courses et le poing sont donc les deux éléments symétriques qui encadrent l'image et lui confèrent son caractère oxymorique et problématique. Le propre de cette illustration est de mettre en tension la figure quotidienne de la ménagère dédiée à ses courses et la figure exceptionnelle du citoyen en colère. Ce contraste est éclairé par le texte qui s'affiche sur les corps des personnages, en réserve blanche : «il est interdit d'interdire de vendre moins cher».

La première partie de la phrase, slogan initial, a été commentée par Jankélévitch (2000, p. 311) : «Cette interdiction est affirmée sans appel, mais l'affirmation de ce veto des vetos échappe elle-même au veto. Exception nécessaire pour que le discours ait un sens... Si elle ne nous est pas accordée, le silence est notre seul recours. Il est interdit d'interdire : vous ne pouvez m'empêcher de le professer, de justifier le droit d'interdire toute interdiction et finalement,

3. Voir [http://expositions.bnf.fr/mai68/grand/042.htm]. 
au nom d'une philosophie dangereusement dogmatique de la liberté, de faire respecter ce droit et, le cas échéant, de réprimer toute infraction au veto des vetos [...]. La liberté nous est donc imposée autoritairement et dans un langage comminatoire propre à intimider les indécis.» (2000, p. 311).

L'interprétation de Jankélévitch évoque le caractère paradoxal de l'assertion tout autant que le risque d'un absolutisme libertaire. Certains peuvent y voir un excès moraliste, d'autres un point de vue logique sur ce qui s'avère être une aporie destinée à frapper les consciences et à inviter à la réflexion sur le bouleversement des valeurs, sur la légitimité des autorités.

Dans le contexte de la mobilisation du slogan par Leclerc, elle devient particulièrement éclairante. Mobilisé par l'enseigne, le slogan est détourné de son usage premier et de sa signification : l'adjonction qui lui est faite détruit son caractère aphoristique. Le slogan devient un argument pour défendre la posture de Leclerc et son combat pour la liberté des prix de la grande distribution et son opposition à la Loi Galland. Loin de constituer un ornement, le détournement du slogan permet d'ancrer le combat politique de Leclerc dans le cadre politique plus vaste et plus ancien de Mai 68. La figure du consommateur se substitue à celle de l'ouvrier; le premier semblant chargé de prendre la relève du flambeau de la contestation à la suite du second. Le combat politique se serait déplacé de la production à la consommation, à l'instar d'une idéologie dans l'air du temps qui affirme l' «empowerment» du consommateur, tendant à valoriser son point de vue critique tout en mettant à distance la puissance des stratégies de marques.

\section{Une stratégie défensive}

Le poing levé devient dans ce contexte discursif, politique, économique et social, un indicateur du combat de Leclerc. Il est aussi l'emblème du glissement qu'opère Leclerc dans sa publicité : la figure d'opposition dans le contexte économique, juridique et politique, c'est Leclerc qui œuvre pour l'abrogation de la loi Galland, connue pour limiter le pouvoir des grandes enseignes. Dans la publicité, le poing levé est celui de la silhouette anonyme d'un consommateur parmi d'autres, prolongation citoyenne et musclée de la ménagère au caddie. En affectant le poing levé au consommateur, Leclerc rallie le consommateur à sa cause, laissant supposer que la lutte est commune. Ce faisant, Leclerc se place en défenseur de la masse des consommateurs, laissant supposer que sa militance pour la baisse des prix n'a pas d'autres objectifs que de satisfaire les consommateurscitoyens. Si rien ne nous permet de douter de l'authenticité du souhait de l'enseigne Leclerc, rien ne permet non plus d'oublier que comme tout acteur économique, Leclerc doit développer une stratégie, assurer sa pérennité en défendant ses marges, affirmer un positionnement cohérent et promouvoir son image pour exister comme acteur économique rentable et pérenne. 
Les autres publicités s’intègrent dans la même perspective avec des modalités expressives différentes.

«La croissance oui sauf celle des prix!»4 : ce slogan s’inscrit dans une posture forte du médiatique patron de Leclerc s'élevant contre l'augmentation des prix décidée par les grands groupes industriels et contre la vision des analystes favorables à une inflation qui allégerait la dette financière.

Un poing fermé se dresse sur ce qui peut être vu comme la cheminée d'une usine, le corps du bâtiment et cette cheminée étant composés d'un assemblage de boîtes de conserves désignées comme telles. Le connaisseur d'affiches de Mai 68 repérera là encore le détournement d'une affiche "La lutte continue» diffusée à l'occasion de l'occupation des usines par les ouvriers. Si la structure de l'image y est la même, la place de la cheminée sur l'affiche y est inversée (volontairement?), à l'instar de l'inversement idéologique autour des perversités ou bienfaits du consumérisme...

Le glissement opère une nouvelle fois; l'usine emblématique de la production se transforme en amoncellement d'une marchandise revendiquant une modération des prix. Si l'auctorialité du «manifeste publicitaire »5 ne laisse aucun doute, le poing fermé semble paradoxalement être ou l'émanation des producteurs (fabricants des conserves) ou l'émanation des supermarchés (mettant à disposition les conserves), ce qui est plus probable. Ce flou témoigne des objectifs conjoints de cette campagne, à la fois destinée à créer une connivence avec les consommateurs et à sensibiliser les politiques (et une presse toujours sensible aux points de vue polémiques) aux postures de Michel Edouard Leclerc dans la défense et la promotion de ses intérêts et de sa vision. Cette publicité est aussi l'affirmation, nécessairement politique, des bienfaits de la croissance, fréquemment mise en cause par ses objecteurs. Mais l'abstraction de la croissance, notion relativement complexe quant à ses modalités et fonctions économiques, se résume ici à l'abondance manifestée par l'amoncellement des boîtes de conserve.

\section{Une lutte contre la défection par l'objectivation du discours}

La défection s'oppose à la prise de parole (Hirschman, 1986, p.75), l'une et l'autre ayant souvent des finalités antagonistes, la défection rendant caduque la prise de parole, celle-ci nécessitant de passer d'une logique silencieuse individuelle à une action collective concertée. Si l'on transpose les propos de l'économiste au cas qui nous préoccupe, on peut analyser la campagne de Leclerc comme le souhait d'échapper à la défection individuelle en investissant la prise de parole.

4. Voir [http://pics.agora.eu.org/spip.php?page=recherche\&recherche=Leclerc].

5. L'expression de «manifeste publicitaire» est empruntée au sémioticien Georges Peninou, qu'il explique notamment dans le chapitre 5 d'Intelligence de la publicité. Étude sémiotique (1972). Une des caractéristiques de ce «manifeste» est de désigner son appartenance au genre publicitaire. 
En effet, dans un contexte de lutte concurrentielle exacerbée en période économique délicate qualifiée de "crise», Leclerc est menacé par les prix bas de ses concurrents et notamment des hard discounters. La masse silencieuse de ses clients peut alors simplement se détourner de la marque enseigne pour accéder aux services des acteurs économiques qui lui sembleront plus attractifs.

La prise de parole n'est pas déléguée par Leclerc aux consommateurs mais son orchestration simule l'action collective en rangeant les consommateurs, du point de vue rhétorique, du côté de l'enseigne qui se désigne comme porteparole. Le poing levé se substituerait dans cette rhétorique à la main invisible chère à Adam Smith, masquant sous l'imagerie l'opacité d'un jeu économique où les équilibres sont précaires, instables et souvent dénoncés comme délétères pour le tissu social (cf. les marges arrière pratiquées par la grande distribution).

En transformant une position stratégique délicate dans le jeu concurrentiel en cause politique de défense des consommateurs, Leclerc lutte contre le risque majeur de la défection de ses clients et tente leur conversion en jouant sur leur fibre contestataire.

\section{Une imagerie captatrice}

Avant l'appropriation du poing levé par les publicitaires, la circulation de ce poing levé dans la culture politique, médiatique et culturelle a contribué à sa «médiagénie » (terme de Philippe Marion) tout autant qu’à son altération, lui faisant perdre parfois en dangerosité politique ce qu'il a gagné en ritualité. L'iconographie des albums d'Astérix et Obélix souligne ce caractère gaulois presque emblématique du «Gaulois moyen », prompt à en découdre mais bon enfant.

La figure du poing levé est donc associée à un jeu connotatif a minima double, à la fois affirmation forte d'une revendication et asepsie de la révolution. L'évocation de Mai 68, à travers le détournement de ses slogans-fétiches, invite à interroger ce double jeu connotatif, l'année 1968 signalée comme celle des « événements» n'étant en général évoquée comme révolution qu'associée au qualificatif «culturelle».

\section{Droits à consommer}

Les révolutions sont prolongées par la mise en place d'un ordre nouveau qui remanie les structures politiques, sociales et juridiques. La Révolution française a ainsi permis la Déclaration des droits de l'homme et du citoyen. Ces droits sont détournés dans la publicité en reprenant la forme de deux grandes générations de droits : les «droits de » et les «droits à », droits sociaux qui impliquent que quelqu'un (l'État en particulier) fasse quelque chose pour vous. La publicité joue principalement sur ce second type de droits centrés sur le bienêtre et donc particulièrement adaptés aux univers à promouvoir des marques. 


\section{Les manifestes : l'affirmation des droits}

À travers trois exemples, celui de l'opérateur de voyage Marmara, d'Yves Rocher et d'Essensis de Danone, nous pouvons observer l'évidage juridique et politique de l'expression «droit à» et sa migration en slogan d'incitation à l'achat. Le «droit à» devient, finalement, «devoir d'acheter» au nom des loisirs ou de la beauté. La légitimité de ce droit est fondée sur une offre commerciale et non plus sur une vision politique et collective. L'évidage juridique converge avec l'emplissage commercial. Les règles, normalement formulées par un organe reconnu légitime par sa portée collective, sont ici élaborées par une marque. La prétention communicationnelle se joue dans le pastiche en inversant les places : le politique passe au second plan, se dissout dans la politique commerciale et la politique de marque s'édifie sur le substrat politique comme instrument de valorisation.

Dans un contexte dit de crise, la marque Marmara, opérateur touristique, a choisi depuis 2006 un slogan fort, «le droit au voyage», comme en témoignent ses diverses campagnes et le rappelle son site Internet : « "Le droit au voyage!" est notre combat au quotidien ». L'opérateur requalifie en besoin le goût de l'évasion et se positionne comme un facilitateur face à cette quête grâce à des prix que le destinataire suppose modérés. Un égalitarisme de bon aloi est implicitement évoqué par le slogan et la marque se dote ainsi d'une qualité morale supplémentaire. Le manifeste publicitaire déplace le champ de la lutte : la lutte concurrentielle, forte sur le secteur du tourisme, est masquée au profit de la mise en avant d'un combat pour les meilleurs prix, au profit du client-voyageur.

\section{Une altération du manifeste politique en manifeste publicitaire}

Yves Rocher, en 2007, fait campagne dans la presse, par un dépliant et parvoie d'affichage avec le slogan «Liberté, égalité, beauté ». La parodie de la devise républicaine est associée à l'imagerie analysée précédemment avec un poing aux ongles vernis de rouge fermé sur une touffe d'herbes (figure 1).

Les trois mots, dans la même typographie, sont dissociés dans la mesure où "beauté», le terme commuté avec le terme absent de «fraternité », est amplifié avec une taille beaucoup plus grande, jusqu'à remplir toute la largeur de l'espace disponible. Les pages intérieures du dépliant développent l'idée d'une beauté féminine fondée sur la naturalité, respect de la nature, celle de la femme et celle de l'environnement.

Le détournement de la devise républicaine évince une fraternité qui, au mieux, aurait pu devenir une «sororité» compte tenu du genre des destinatrices, au profit d'une beauté érigée en valeur fondamentale dans le monde Yves Rocher. Par ailleurs, la fraternité du point de vue du consumérisme constitue une valeur beaucoup moins porteuse qu'une liberté flatteuse pour l'individualisme 


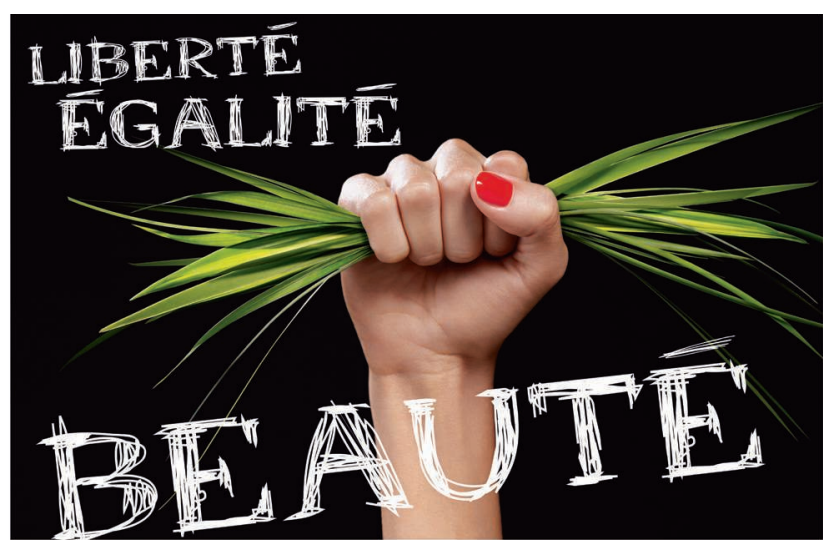

Figure 1. Campagne Yves Rocher (C) 2007

et qu'une égalité judicieuse permettant à chacune de revendiquer sa part de beauté. Cette beauté est d'autant plus égalitaire et raisonnable qu'Yves Rocher mise sur une "esthétique de l'intérieur» et une harmonie esthétique fondée sur le respect de la nature (acceptation du vieillissement, éthique), la joie d'être naturelle associée aux produits de la marque jouant le rôle de philtre de beauté.

\section{Affirmer la rupture pour se positionner}

C'est un autre jeu de valeurs que proposa la marque Essensis de Danone lorsqu'elle se lança sur le marché en 2007 avant de disparaître en 2009. Première marque «alicament », le yaourt Essensis se positionnait comme un produit de beauté alimentaire. Si la composition du produit n'est pas nécessairement un bouleversement en soi, le positionnement revendiqué par la marque d'alicament sembla légitimer une communication de rupture, à la hauteur de l'ambition.

En toute cohérence, la promotion de la marque se fit ainsi en partie grâce à l'impression et la diffusion dans la presse d'un livret fuchsia (couleur du packaging du produit) affirmant sur sa «Une» : «Déclaration universelle des droits de ma peau». Si le possessif relativise quelque peu le caractère universel de cette déclaration, le détournement se déploie avec la production d'un «préambule » pastiché. Le ton est humoristique : «Considérant l'importance de l'enjeu, l'Assemblée Générale présidée par Moi [...] proclame la Déclaration Universelle des droits de ma peau ». Ce préambule est suivi de quinze articles - de l'art. $1^{\mathrm{er}}$, «Toutes les peaux naissent et demeurent libres et égales en droit », à l'art. 15, «Nous nous engageons à favoriser la diffusion et la lecture de la déclaration universelle des droits de ma peau », sans oublier, à l'art. 13, la création d'un «ministre des caresses » afin de "guider les peaux amies vers un avenir plus radieux». Les articles sont illustrés avec des photos de femmes montrant largement leur peau, 
visage et corps nus en postures diverses : se nourrissant, s'arrosant, levant leur poing enserrant le livret rose. Les articles sont suivis de quelques pages commerciales reprenant la rhétorique publicitaire traditionnelle de la cosmétique largement fondée sur la valorisation scientifique (schéma en coupe des strates de la peau avec « efficacité prouvée», composition du produit, etc.).

La marque se positionne sur l'idée d'une rupture entre un avant du produit et un après, la révolution correspondant à la mise sur le marché d'Essensis. En ce sens, la communication porte en cohérence l'affirmation d'une innovation «révolutionnaire ». La forme de la Déclaration des droits de l'homme et du citoyen sert ici de matrice au développement d'arguments aussi variés que l'affirmation de cette innovation-révolution, les ingrédients du produit, les bénéfices promis, l'activation du bouche-à-oreille entre clientes.

Pour autant, là encore, le régime euphorique du discours, la couleur du dépliant, le caractère individualiste et intime contrastent avec les caractéristiques du modèle fondateur solennel, collectif et public.

\section{Les «Droits», occasion d'un remaniement énonciatif}

Le choix générique opéré par les marques, même si elles opèrent dans un «comme si » propre à la publicité comme genre métadiscursif, témoigne de leur ambition communicationnelle et de leur envie de dire les lois, c'est-à-dire d'affirmer comme norme une vision du monde.

Cette ambition pourrait être reçue d'emblée comme suspecte, mais elle est euphémisée dans le genre même qui la soutient. En effet, à l'instar de la Déclaration des Droits de l'Homme qui s'analyse comme un «énoncé d'une modalité déontique tout en se présentant comme un énoncé non modalisé » (Martin, 2010), les déclarations observées produisent des énoncés avec une tendance au «flou référentiel» (Adam, Bonhomme, 2000, p.47), normatifs, prescriptifs, centrés sur un apparent bien commun.

Les slogans sont porteurs d'une modalité déontique c'est-à-dire qu'ils conduisent à une morale avec les idées de devoir, de nécessité. Dans le contexte, il semblerait qu'ils soient avant tout porteurs d'une permission qualifiée généralement, dans la terminologie du marketing, de « levée des freins » à l'achat.

En énonçant de cette façon, les Déclarations des marques prescrivent la norme sans avoir l'air d'y toucher, en se disculpant d'un trop ostensible intérêt. Par la force formelle de l'emprunt révolutionnaire, elles luttent dans le même temps contre la fadeur du conformisme, une certaine mollesse autour de notions consensuelles et de consommations a priori non vitales (les voyages, la beauté....).

Basculant entre texte descriptif et texte prescriptif, la publicité permet aux marques de jouer sur le cadre énonciatif qu'elles adoptent et d'accroître la valeur symbolique de leurs offres. 
Au passage, elles transforment leur statut : en s'arrogeant le droit de dire les lois, elles proclament leur légitimité sur le secteur d'activité qu'elles promeuvent et se présentent en leader face à leurs concurrents potentiels. Les Déclarations sont des déclarations de guerre concurrentielles, elles énoncent un affranchissement de ce jeu de la concurrence pour asséner aux autres leur propre autorité.

Les Déclarations se réfèrent ainsi à deux modalisations du discours politique, à la fois didactique et polémique. Leur ancrage "révolutionnaire » leur confère une modalité supplémentaire : le contrôle du temps. En affirmant un "droit à », les marques entérinent une rupture par rapport au passé et reconfigurent ainsi un système propre.

Ces exemples de détournement et d'intertextualité illustrent la façon dont le sens se déploie dans l'affirmation des droits, évide l'affirmation politique profonde pour l'utiliser comme matrice rhétorique propre à créer de la valeur symbolique.

\section{L’idéal révolutionnaire comme réservoir de puissance}

\section{Le caractère performatif des formules révolutionnaires}

Les discours et rituels politiques ont une forte valeur performative que l'on retrouve ici appropriée par le discours publicitaire.

Les énonciations déclaratoires dont usent Marmara, Yves Rocher et Essensis sont éminemment performatives et la caractéristique de ces Déclarations, comme de tous les performatifs, n'est pas d'être vraie ou fausse mais d'être agissante.

Les formules révolutionnaires rejoignent ainsi l'idéal publicitaire, celui d'actualiser le discours en acte, d'assimiler la formulation d'un discours à sa virtualité opératoire, de transformer l'objectif de communication en réalité marchande, de muer le destinataire en consommateur. Les slogans publicitaires ne sont-ils pas destinés, à l'instar des slogans politiques, à être motivants, propres à mouvoir?

La valeur iconique du poing levé est forte car son caractère puissamment phatique (il exprime une émotion forte destinée à interpeller l'extérieur) se double d'un caractère performatif : «dire, c'est faire» (Austin, 1970) et le fait même d'exprimer sa colère et revendication en levant le poing, c'est faire exister cette contestation, la rendre active. La mobilisation du consommateur est rendue visible, le manifeste publicitaire actualise un phénomène social et politique possible. Leclerc fait migrer cette probabilité sociale vers sa cause et canalise le phénomène à son profit.

La connaissance, par l'ensemble des Français, de l'existence d'un passé politique français marqué par des révolutions, mouvements politiques, manifestations, "événements» atteste de la crédibilité de certaines formes 
iconiques et discursives : cela s'est passé, cela a existé et par extension, cela inscrit la globalité du manifeste publicitaire dans un horizon crédible.

\section{Les figures de consommateurs-citoyens}

Les cas analysés illustrent la valorisation d'une dimension particulière du consommateur : sa capacité à protester et à se rebeller. Certes, c'est une rébellion qui n'est guère effrayante car elle apparaît quelque peu édulcorée par le régime discursif publicitaire au singulier, la foule, et sa dangerosité virtuelle, étant absente. La contestation est toutefois suffisamment spectaculaire et phatique pour s'imposer comme l'affirmation d'un équilibre des rapports entre consommateurs et entreprises. Cette représentation actuellement très en vogue, reprise à la fois dans les stratégies publicitaires et les stratégies de marque dites «hors média » telles que la production des contenus, la «conversation » sur les réseaux sociaux et autres tactiques communicationnelles, dénie les stratégies captatrices des marques en général et de la publicité en particulier (de Montety, Patrin-Leclere, 2011).

À travers cette figure spectaculaire de l'engagement politique, les acteurs du marketing et de la publicité tendent à «élargir les préférences du consommateur aux valeurs citoyennes » et donc à " politiser le marché». Quant aux producteurs, dans certains cas comme celui des grandes enseignes, «ils s'emparent de la politique pour redéfinir les relations de production » (Cochoy, 2008, p.110). L'auteur établit une comparaison aussi audacieuse qu'heuristique entre les dispositifs de l'isoloir et du chariot, les deux produisant des effets politiques mais l'un fabrique « un sujet isolé décidant seul en le “coupant” de la société; d'un autre le chariot réagrège un collectif» (id., p. 118). Cette conception qui tend à assimiler le marché à l'arène politique n'est qu'en partie celle des publicitaires qui se saisissent du politique car ils passent nécessairement sous silence la question pourtant vive du «vouloir d'achat » posée notamment par les emblématiques décroissants qui grossissent les rangs des antipubs.

\section{Une éviction de l'idéal politique?}

Dans un travail antérieur (Souchier, 1992, p.50), l'analyse fine de l'énonciation de campagnes publicitaires de Volkswagen détournant le drapeau soviétique conclut au détournement comme traduction du sens politique d'une époque, celle du «triomphe de l'économie face au déclin de ce qui fut une lutte pour un idéal».

On peut légitimement émettre l'hypothèse de la transformation du politique sous l'influence des représentations qui lui sont associées, la publicité jouant un rôle de propagateur, contribuant à rendre visible ou à taire, altérant et recréant inlassablement... 
Pour autant, la publicité désigne plus certainement les représentations de ses médiateurs que celles de la société. Ces campagnes, polysémiques, s'adressent à des destinataires qui peuvent adhérer, ou pas, de diverses manières au manifeste publicitaire : adhésion de fond au message, sensibilité à l'habileté rhétorique, plaisir esthétique, désaveu politique, indifférence... Le message n'engendre pas plus le consommateur que le citoyen même s'il peut, à la marge, transformer des représentations existantes.

\section{La politique mythifiée}

Le discours politique est mis à l'honneur dans les campagnes fondées sur la valorisation de la contestation et de la révolution. Mais dans cette appropriation, le commerce devenant le lieu même de l'expression politique, l'idéal politique passe finalement au second plan.

Ce brouillage des champs de la vie sociale est une caractéristique très fréquente et de plus en plus puissante de la rhétorique publicitaire qui n'hésite pas à se saisir de champs sociaux et grands thèmes anthropologiques longtemps préservés, voire intouchables (politique, religion...) quand ces champs sont mis à distance et évoqués non plus pour stimuler un croire et un engagement mais comme substances culturelles de nos répertoires (Barthes) ou encyclopédies (Eco) collectives. Comme on l'a vu dans la mobilisation des formules et imageries révolutionnaires, grâce à la représentation qui met «la force en réserve dans les signes» (Marin, 1981), ces substances sont porteuses d'une force très intéressante pour le système rhétorique publicitaire. Il peut tisser son discours en mobilisant une réserve de signes et de discours susceptibles d'être utilisés comme matrices de transformation d'ambitions marchandes en promesses de consommation.

La révolution, notion forte, constitutive de notre histoire politique, sociale et culturelle, représentation puissante et élevée associée aux idéaux collectifs, devient ainsi, dans le contexte publicitaire de subtilisation de substance, une forme-écrin consensuelle pour dire son rapport à la consommation, valoriser des stratégies de marque et y enchâsser des promesses de consommation à destination de consommateurs appréhendés de façon holistique. Cette forme c'est celle du mythe, processus de renversement de valeurs, évoqué en introduction et présent en filigrane des analyses présentées. Pour Barthes, « les hommes ne sont pas avec le mythe dans un rapport de vérité mais d'usage : ils dépolitisent selon leurs besoins. [...] Il suffit de modifier ses entours, le système général (et précaire) dans lequel il prend place, pour régler au plus près sa portée» (Barthes, 1957, p. 232-233).

La mythification des discours et imaginaires du politique traduit leur force sociale, illustre leur succès et leur diffusion, mais témoigne aussi de leur altération, leurs valeurs étant transformées lors de leur circulation (Jeanneret, 2008). 
Cette transformation de valeur est activée dans le champ des médiations marchandes par la consommativité, définie comme «valeur des objets corrélée à leurvalorisation sociale» (Baudrillard, 1972, p. 87).

La mobilisation de ce mythe est à la fois révélatrice de la puissance des idéaux qu'il charrie, car la publicité ne saurait se saisir de ce qui lui semble sans valeur, et dans le même temps cette mobilisation mine, par sa seule évocation hors contexte, la valeur première et fondamentale de ce qui a été confisqué.

Nous avons observé dans les campagnes analysées la façon dont la révolution, emblématique de la grandeur du politique, est mobilisée comme mythe. Si cela traduit sa force sociale, le geste publicitaire entraîne une certaine asepsie de la révolution avec le gommage des conditions matérielles et sociales de son accomplissement et son exaltation en spectacle consensuel à consommer. Le caractère performatif des énoncés révolutionnaires induit un passage à l'action; dans le contexte publicitaire, c'est un passage à l'acte social corrélé à l'acte d'achat, valorisé comme euphorique dans cette double proposition. Il correspondrait, dans une perspective narratologique, à une « sanction » positive : une récompense, l'aboutissement heureux du récit qui a précédé. Dans les campagnes analysées, l'achat équivaut à une avancée sociale : audelà de la consommation individuelle, il est évoqué comme acte vertueux pour la société tout entière.

Cette instrumentation dépolitise la révolution même si elle proclame sa valeur sociale. Certains déploreront que la publicité substitue aux idéaux politiques des aspirations consuméristes. En effet, quand la publicité mobilise le motif révolutionnaire pour transcender la portée sociale des offres marchandes, elle évide la révolution de sa dimension politique en feignant d'affirmer ou en affirmant des attentes citoyennes que la société de consommation viendrait combler. Mais les citoyens sont-ils dupes de cette rhétorique, la redondance de la trame narrative la désignant comme syntagme figé, les publicités se chargeant pour l'alimenter de tous les thèmes et valeurs disponibles dans l'espace culturel, pour peu qu'ils soient potentiellement valorisants pour les offres à promouvoir?

N’oublions pas par ailleurs que la publicité se désigne elle-même, dans les cas présentés, comme rhétorique de la consommation : les campagnes diffusées par affiches ou presse, supports légitimes de la publicité, s'adressent à des consommateurs contemporains sensibilisés à la communication marchande, aptes à qualifier le contexte énonciatif des messages, leur intention. On peut du coup faire l'hypothèse de leur capacité à relativiser l'adresse citoyenne qui leur est faite tout autant que l'irrévérence manifestée à l'égard de l'idéal révolutionnaire... Une énonciation assumée dans un cadre clairement publicitaire, ce qui n'est pas toujours le cas mais l'est dans le cadre des campagnes analysées, élimine ou amoindrit le risque de confusion entre l'idéal-type de la révo- 
lution et sa circulation dans l'espace social. La révolution dans ces publicités est bien un motif, prétexte pour construire une rhétorique marchande.

Toute révolution configure et signifie un basculement de valeurs et un ordre nouveau. S'en saisissant, la rhétorique publicitaire valorise la posture des marques et enseignes, confère une aspérité à leurs offres prétendument « révolutionnaires» tout autant qu'elle affirme un équilibre des relations entre producteurs et consommateurs, ralliés à un idéal commun. Au passage, le procédé discursif désigne les fondements mêmes de la publicité car, en se saisissant du motif révolutionnaire, la publicité opère une mise en abîme de son propre fonctionnement et de son ambition : promouvoir des passages à l'acte en les corrélant à des valeurs, légitimer une vision du monde et proclamer un ordre nouveau.

\section{Références}

Adam Jean-Michel, Bonнomme Marc, 2000, L'argumentation publicitaire. Rhétorique de l'éloge et de la persuasion, Paris, Nathan Université.

Austın John L., 1991 [1970], Quand dire c'est faire, Paris, Le Seuil (Points).

BArthes Roland, 1970 [1957], Mythologies, Paris, Le Seuil (Points).

Baudrillard Jean, 1976 [1972], Pour une critique de l'économie politique du signe, Paris, Gallimard (NRF).

Berthelot-Guiet Karine, Montety Caroline (de), 2009, "Hyperpublicitarisation et dépublicitarisation. Métamorphoses du discours des marques et gestion sémiotique», La Publicité d'aujourd'hui. Discours, formes et pratiques, Y. Lebtahi, F. Minot éd., Circav nº 20, Paris, L’Harmattan, p. 63-77.

Cochor Franck, 2008, "Faut-il abandonner la politique aux marchés? Réflexions autour de la consommation engagée », Revue française de socio-économie, n⿳01, p.107-129.

Habermas Jürgen, 2007 [1963], L'espace public. Archéologie de la publicité comme dimension constitutive de la société bourgeoise, Paris, Payot.

HIRSCHMAN Albert O., 1986, "Défection et prise de parole», Vers une économie politique élargie, Paris, Minuit.

JAN KÉLÉVITCH Vladimir, 2000, «L'ambiguïté morale en son for intérieur», Philosopher, $n^{0} 1$, C. Delacampagne, R. Maggiori éd., Paris, Fayard.

JeAnNeret Yves, 2008, Penser la trivialité, vol. I : La vie triviale des êtres culturels, Paris, Hermès, Lavoisier.

MARTIN Fabienne, 2010, «Indéfini, modalité et généricité dans la Déclaration des droits de l'homme», Argumentation et analyse du discours, en ligne [http://aad.revues. org/770], consulté le 7 mai 2011.

MARIN Louis, 1981, Le portrait du roi, Paris, Minuit.

Montety Caroline (de), PATRIN-LeCLere Valérie, 2011, "La conversation. Le succès d’un succédané », Communication et langages, nº169, $4^{\mathrm{e}}$ trimestre, p. 23-37. 
Peninou Georges, 1972, Intelligence de la publicité. Étude sémiotique, Paris, Robert Laffont.

SOUCHIER Emmanuel, 1992, "La publicité comme détournement du politique», Communication et Langages, $\mathrm{n}^{\circ}$ 93, $3^{\mathrm{e}}$ trimestre, p. 36-51.

VERGNON Gilles, 2005, "Le "poing levé”, du rite soldatique au rite de masse. Jalons pour l'histoire d'un rite politique », Annales, n³, mai-juin. 